\title{
Schmerz-Petition 2011
}

\author{
Ärzte fordern bessere Verfügbarkeit innovativer Medikamente
}

Zwar stehen für sehr starke chronische Schmerzen heute bereits gute therapeutische Möglichkeiten zur Verfügung, in Österreich ist der Zugang zu diesen innovativen Medikamenten jedoch mit einigen Hürden verbunden. Einige medizinische Fachgesellschaften machten daher kürzlich mit einer Schmerz-Petition auf dieses Problem aufmerksam. Sie stellen darin fest, dass zusätzliche innovative Schmerztherapeutika zur Behandlung chronischer Schmerzen notwendig sind und fordern von der Sozialversicherung, dass die Therapieempfehlungen der Experten berücksichtigt werden.

„Die Petition warnt, dass wir in Österreich bereits in Verzug geraten sind“, stellte Univ.-Doz. Dr. Sibylle Kozek-Langenecker, Präsidentin der Österreichischen Gesellschaft für Anästhesiologie, Reanimation und Intensivmedizin (ÖGARI) anlässlich der Präsentation der Initiative Ende Oktober in Wien fest: „Hochwirksame Medikamente zur Schmerztherapie, die in der EU zugelassen sind, stehen in Österreich nicht im Erstattungskodex." Während der Zugang zu diesen Medikamenten im stationären Bereich aufgrund des anderen Finanzierungssystems, offen ist, bedeutet dies für den Patienten in der ambulanten Behandlung einen mühsamen Weg der chefärztlichen Bewilligung und in vielen Fällen eine unüberwindbare Hürde. Denn, so Kozek-Langenecker: „Was nicht erstattet wird, ist de facto nicht verschreibbar und nicht vorhanden." Ein Umstand, der auch bei den behandelnden Ärzten für Frustration sorgt, wenn nach einer langwierigen Diagnostik ein maßgeschneider- tes multimodales Konzept erarbeitet wurde und ein wesentlicher Bestandteil in der Praxis nicht einsetzbar ist, „,weil wir an pseudoökonomische Limitierungen stoßen." Denn langfristig verursacht die scheinbar ökonomischere Therapie wesentlich höhere Folgekosten - durch Depressionen aufgrund unzureichender Schmerzreduktion, schweren Nebenwirkungen der konventionellen Medikamente, die höher dosiert werden müssen, und häufigere Arzt- und Spitalsbesuche.

\section{Schmerz-Petition 2011}

- Als Ärztin/Arzt kann ich aufgrund meiner Erfahrung im täglichen Umgang mit Schmerzpatienten bestätigen, dass zusätzliche innovative Schmerztherapeutika notwendig sind.

- Klinische Therapieempfehlungen von Fachgesellschaften in Österreich sollen auch von der Sozialversicherung adäquat respektiert und umgesetzt werden.

Vier österreichische Fachgesellschaften - Anästhesie, Reanimation und Intensivmedizin, Schmerz, Palliativmedizin und Psychiatrie und Psychotherapie riefen im vergangenen Sommer österreichweit Schmerztherapeuten im ambulanten und stationären Bereich zur Unterstützung der Ärzte-Schmerz-Petition auf. Mit knapp 1.400 Unterschriften wurde sie Anfang Oktober an den Hauptverband der Sozialversicherungen übergeben. Nun soll in einer gemeinsamen
Arbeitsgruppe eine für alle Seiten vertretbare Lösung gefunden werden.

1,7 Millionen Betroffene in Österreich mit einem Anteil von 21 Prozent, die aufgrund der chronischen Schmerzen unter Depressionen leiden, 20 Prozent, die ihren Job wegen der schweren Schmerzen verloren haben, durchschnittlich 15 verlorene Arbeitstage durch Schmerz pro Jahr zeigen den Bedarf für eine verbesserte Schmerztherapie auf. „Wir wollen unsere Patienten wirksam, schonend und zielgerichtet behandeln“, unterstrich Univ.-Prof. Dr. Wilfried Ilias, Vorstand der Abteilung für Anästhesiologie und Intensivmedizin, Krankenhaus der Barmherzigen Brüder in Wien. Dazu müsse es auch möglich sein, alle derzeit verfügbaren Therapien zum Wohle des Patienten tatsächlich einsetzen zu können. Die knappe Finanzsituation der Krankenkassen könne nicht auf Kosten des Patienten gehen. Es gehe nicht um einen undifferenzierten breiten Einsatz, sondern um die individuelle Abstimmung mit modernen Therapiekonzepten. Im ambulanten Bereich stehen beispielsweise zur Behandlung von Durchbruchschmerzen die innovativen transmukosalen Fentanylpräparate nicht zur Verfügung. Der Patient muss stattdessen auf ein injizierbares, länger wirksames Produkt zurückgreifen, das bei jeder Anwendung die Gefahr einer Infektion birgt, erklärte Ilias, und darüberhinaus die Aktivität des Patienten während der Dauer der Wirksamkeit massiv einschränkt. Ziel der Petition ist daher: Die Lebensqualität der Betroffenen zu verbessern und trotzdem das Budget nicht zu überlasten. 